\title{
Aerodynamic Effects of Blade Positive Sweep in Axial Flow Cascades
}

\author{
Ali R. Kwedikha*, Abdul Azeam A. Elgayed, Abelmalek N. Algaaoud \\ Department of Mechanical Eng., College of Engineering, University of Zawia, Libya \\ DOI: https://doi.org/10.21467/proceedings.4.16 \\ * Corresponding author email: ali.kwedikha@zu.edu.ly
}

\begin{abstract}
In this work, the effects of sweep applied to rotor of axial flow turbomachines were investigated by means of applying computational fluid dynamics (CFD) tools. Lowaspect-ratio rotors of positive sweep (PSW) have been studied and compared to unswept datum (USW) rotors, at one flow rate and different spanwise locations.

Comparative studies have been carried out on positive sweep and upswept rotors at the design flow rate, by means of developing structured fully hexahedral mesh of the entire computational domain. The structured meshing technique offers the moderation of cell number and skewness, and makes possible cost-effective CFD investigations. Investigations of inlet and outlet flows field were carried out at the design flow rate.

It was pointed out that the efficiency for the positive sweep rotor is deteriorated near the tip and near the hub at the design point.
\end{abstract}

Keywords: Three-dimensional turbomachinery flow, blade sweep, structure meshing.

\section{Introduction}

In the turbomachinery manner, blade positive sweep may possibly give opportunity to control the flow and to demonstrate the impact of blade positivity on rotor aerodynamics, thus contributing to a more comprehensive understanding of the effects of consequences. This paper presents an investigation using computer codes for studying the blade sweep positivity effects on the Three-Dimensional (3-D) flow of axial fan.

A comparative investigation using a computational Fluid Dynamics (CFD) tool for Positive Sweep (PSW) and Unswept (USW) blades is carried out. Figure 1 shows the front views of these blades.

Low-aspect-ratio rotor of PSW sweep has been studied and compared to unswept datum rotor, at the design flow rate and different spanwise locations. Sweep is a non-radial blade stacking technique. Sweep is forward or backward if a blade section at a given radius is upstream or downstream of the adjacent blade section at lower radius respectively, and termed positive or negative if a blade section under consideration is upstream or downstream of the adjacent inboard blade section, respectively [1].

(C) 2018 Copyright held by the author(s). Published by AIJR Publisher in Proceedings of First Conference for Engineering

Sciences and Technology (CEST-2018), September 25-27, 2018, vol. 2 .
This is an open access article under Creative Commons Attribution-NonCommercial 4.0 International (CC BY-NC 4.0)

AiR license, which permits any non-commercial use, distribution, adaptation, and reproduction in any medium, as long as the original work is properly cited. ISBN: 978-81-936820-6-7 
In the literature, several studies have been carried out for axial flow turbomachinery in order to investigate the effect of backward or forward sweep on the 3-D flow of axial fan. These studies have focused on the interblade and downstream flow phenomena e. g. Yamaguchi et al. [2]. Others report negligible effects of pure positive sweep on the upstream flow field, as in Clemen et al. [3]. Few researches focused on the pure positive sweep of the turbomachinery rotors. Therefore, lack of information appears in the literature concerning positive sweep rotor designs. This has made some difficulty to compare with the results of other research projects. According to Braembussche and Vad [1], no generally valid concept for aerodynamically optimum prescription of blade sweep angle along the span has been published in the open literature.

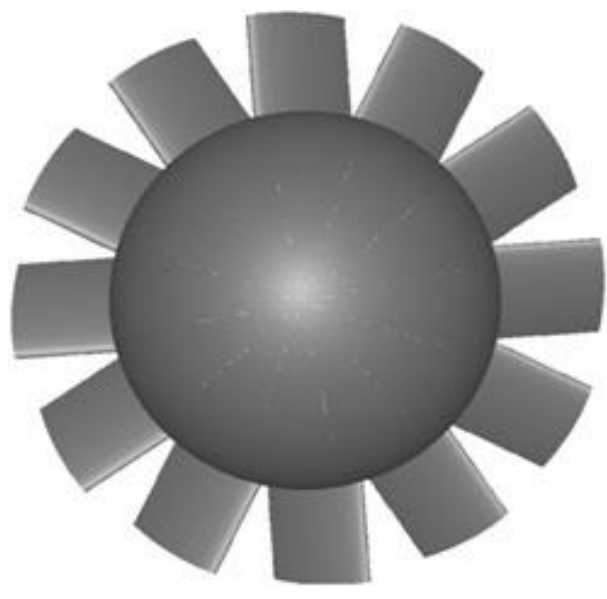

USW rotor

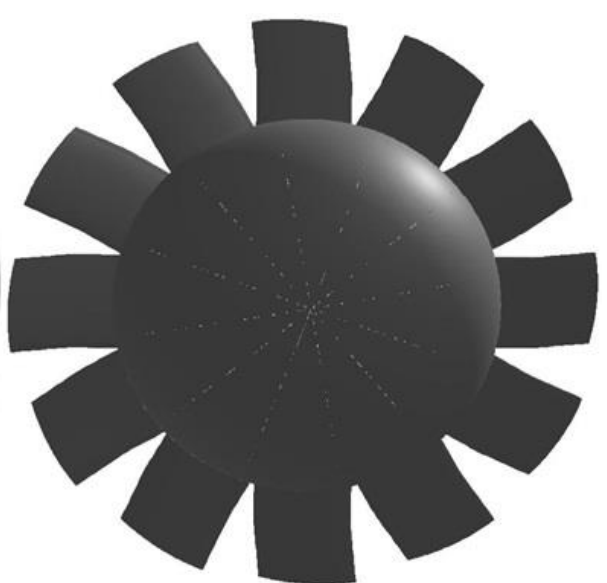

PSW rotor

Fig. 1. Front views of USWand PSW rotors

The study presented here contributes to a more comprehensive understanding of the positive sweep effects. The aim is to provide some answers not presented by the available literature. This study provides a comparative study between USW and PSW rotors using CFD tools. The numerical simulation was carried out using ANSYS Student Release 17.2, the code is a state-of-the-art computer programs for modelling fluid flow in complex geometries, using Finite Volume Method (FVM).

Some assumption used in this work such as: the flow is considered steady state, incompressible, with swirl-free inlet, no entrance guide vane and negligible heat transfer.

\section{Blade positive sweep geometry}

The stacking line (SL) is the line passing through the centres of gravity of the blade airfoil sections enclosed in cylindrical stream tubes. The chord line is the straight line connecting the two points of leading edge (LE) and trailing edge (TE) of each blade section. Blade sweep is known as technique of non-radial blade stacking. Sweep can be provided if the blade sections 
Aerodynamic Effects of Blade Positive Sweep in Axial Flow Cascades

of a datum blade of radial stacking line (RS) are displaced parallel to the chord [4] as sketched in Figure 2a. A blade is swept forward (FSW) or backward (BSW) at a given radius if the blade sections of a radially stacked datum blade are shifted parallel to their chord in such a way that a blade section under consideration is upstream or downstream of the neighbouring blade section at lower radius, respectively [5], Sweep is said to be positive near an endwall when a blade section under consideration is upstream of the adjacent inboard section, as shown in Figure2.

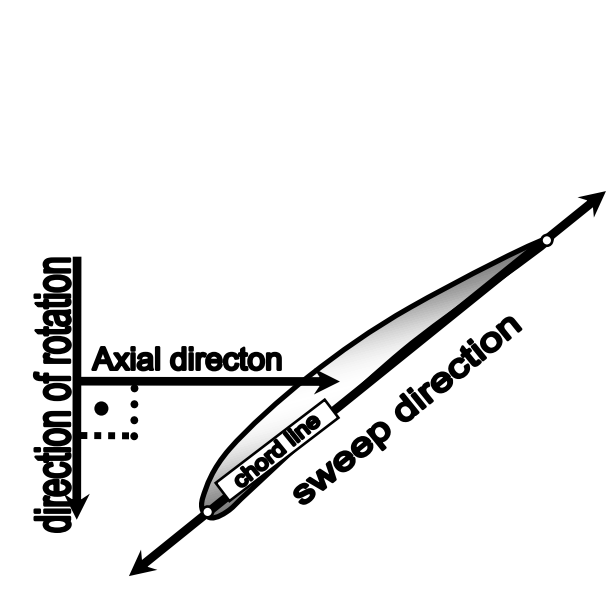

a) Direction of sweep

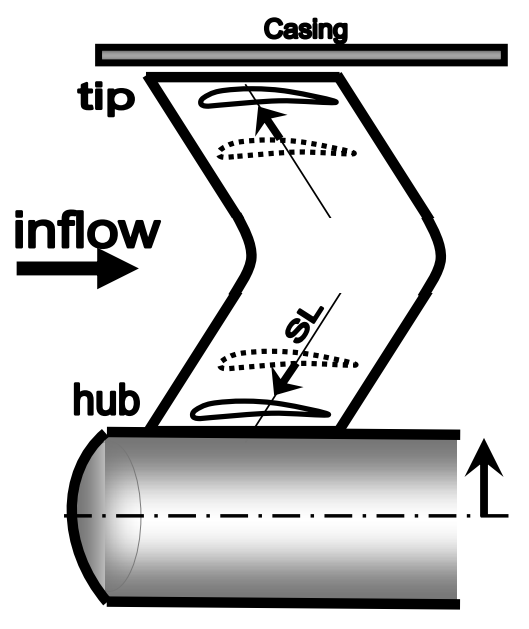

b) Schematic drawing for PSW

Fig. 2. Sweep direction and schematic drawing for PSW

In this case study, reference [6] is taking as preliminary reference. The rotor blade sections has C4 (10\%) profile along the entire span, with circular arc camber lines. The Reynolds number $(\mathrm{Re})$ determined using speed of blade tip, characteristic length of chord tip and the kinematical viscosity of air at $20^{\circ} \mathrm{C}$ is approx. $1.074 \times 10^{6}$.

Taking the reference velocity $\left(\mathrm{u}_{\mathrm{ref}}\right)$ as the tangential velocity of the blade tip $\left(\mathrm{u}_{t}\right)$, the global flow coefficient $\left(\Phi_{\mathrm{D}}\right)$ is defined as the area-averaged axial velocity in the annulus divided by $u_{\text {ref. }}$ Hub-to-tip ratio ( $v$ ) is defined as the ratio of blade hub diameter to the blade tip diameter, while the tip clearance $(\tau)$ is defined as the ratio of rotor tip clearance to the blade span. The main fan characteristics are summarised in Table 1.

Table 1. Main fan characteristics[6]

\begin{tabular}{||l|c|}
\hline Casing diameter & $2000 \mathrm{~mm}$ \\
\hline Hub-to-tip ratio $\mathrm{v}$ & 0.6 \\
\hline Rotor blade count $\mathrm{N}$ & 12 \\
\hline tip clearance $\tau$ (\%span) & 5 \\
\hline flow coefficient $\Phi_{\mathrm{D}}$ & 0.33 \\
\hline
\end{tabular}


The rotor blades are arranged in such a way that, the blades are assembled in an annular cascade with cylindrical and part of spherical as well as cylindrical casing, as shown in Figure3.
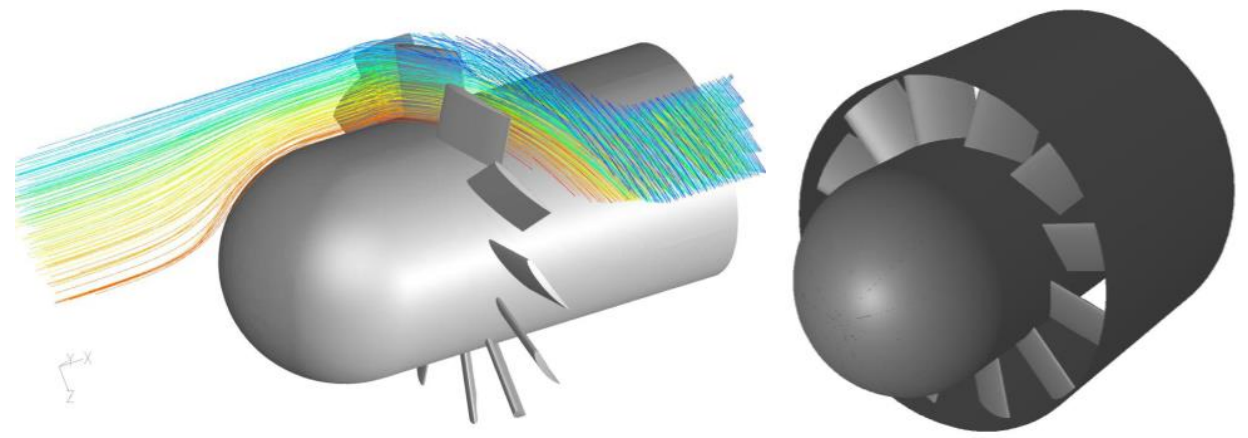

Fig. 3. Virtual isometric of USW rotor

In this study, to create positive sweep the blade sections are swept upstreamlly from the middle of the blade span to the both endwalls by 3.5 degree.

\section{CFD technique}

The CFD investigations were carried out by using the commercial available finite-volume ANSYS-17.2 code. 3-D geometry was constructed by split volumes and meshed using multiblocks structured grids. This technique used to provide hexahedral meshes for the whole geometry of both rotors. It must be noted as Wenneker stated that, discretization of the flow equations on unstructured grids is considered to be more difficult than on structured grids [7]. 2-D structured meshes take place in two essential forms $\mathrm{H}$-grids and C-grids. With consideration of periodicity, one computation domain instead of all geometry has been created to avoid the mesh size and time consuming. Typical computational domain for USW rotor is presented in Figure 4.

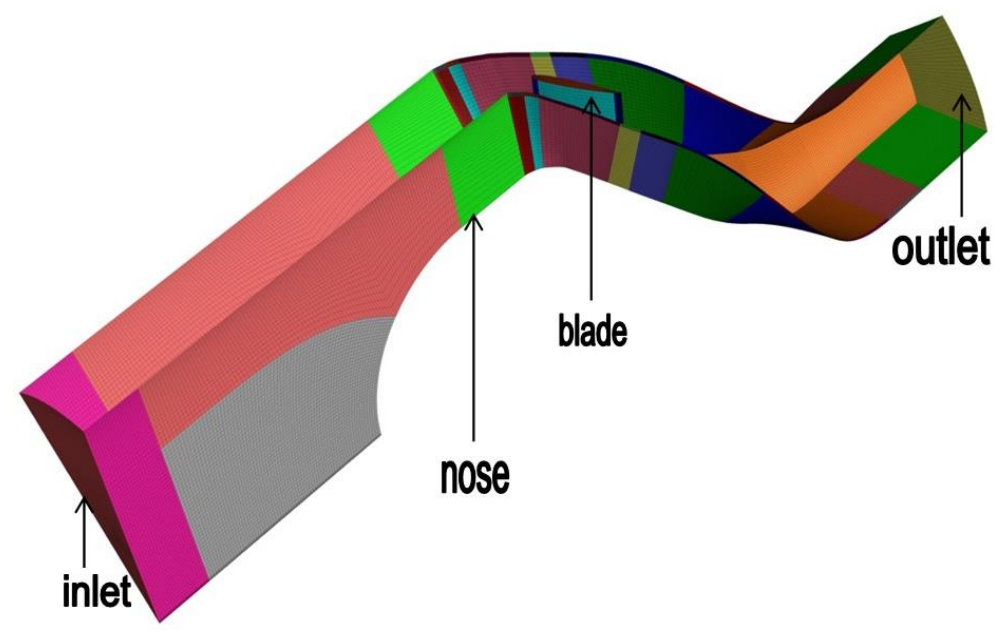

Fig. 4. Typical computational domain for USW rotor

Proceedings of First Conference for Engineering Sciences and Technology (CEST-2018), vol. 2 
Aerodynamic Effects of Blade Positive Sweep in Axial Flow Cascades

The domains extend to approx. 7 and 4 midspan axial chord lengths in the axial direction upstream and downstream of the rotor blading, respectively. The coordinates of domain in $\mathrm{x}$ axis started from (-1750 to $2000 \mathrm{~mm}$ ). Sector shape is inlet face with 30 degree central angle. Cone and hub sectors with one blade in the middle of the domain are provided next to the downstream of inlet face.

\subsection{Boundary Conditions}

- Inlet boundary condition: the inlet is set as a velocity inlet with magnitude of 9.2 $[\mathrm{m} / \mathrm{s}]$. The flow direction is parallel to the rotational $\mathrm{x}$-axis.

- Wall boundary condition: the blade, hub and outer cassing have been defined as wall.

- Outlet boundary condition: outflow is used for the condition of outlet boundary.

- Interior boundary condition: the remaining part of the domain is set as default interior and the air fluid is applied with density of $1.225 \mathrm{~kg} / \mathrm{m}^{3}$.

- Periodic condition: all the side surfaces of the domain are defined as rotational periodic.

The standard $k-\varepsilon$ model with enhanced wall treatment has been used as the turbulence model. The nondimensional wall normal cell size y+ values mostly fell within the range of 30 to 100, which agreeable with the requirements of the applied law of the wall. The discretisation of the convective momentum and turbulent quantity fluxes were carried out for both rotors by the second Order Upwind method. Typical computations required approximately 5000 iterations. The solutions were considered converged when the scaled residuals reached to $10^{-8}$.

\subsection{Meshing}

To provide hexahedral meshes, the domain has been spitted to 31 volumes for both rotors and meshed using multiblocks structured grids. Biswas and Strawn stated that, hexahedral meshes provide a more accurate solution than their tetrahedral counterparts for the same number of edges [8], in this work; all the volumes have been meshed by the method of "Cooper". The most of the domain was meshed with "Hexahedral" structured meshing. Before starting with 3-D meshing, two types of grid meshing are applied in 2-D topology, Ctype meshing applied for the vicinity of blade LE and TE, whereas H-type applied in most of the left. Dense meshing near the blade LE and TE as well as near the hub and tip is applied as in work of Kamenik et al [9]. The structured grid is usually applied to a relatively simple configuration. However, the swept blade geometry is being considered as a relatively complex design, in which a result of applying 3-D annular cascade instead of linear cascade. The total number of cells for USW and PSW rotor are 498889 and 469410 hexahedral cells, respectively. To develop annular cascade configuration and to take advantages of the periodicity, boundary condition of periodicity for one domain was applied, so that each meshing points and arrangement of each periodic face were created just identical with corresponding matching 
face. Acceptable equiangle skewness of cell volume was achieved; the maximum skewness values are 0.82 and 0.75 for $\mathbf{U S W}$ and PSW rotors, respectively.

\section{Results and Discussion}

\subsection{Comparative flow survey of PSW and USW rotors at design flow rate}

Results of valuable analysis by means of CFD technique for a comparison between PSW and USW rotors can be achieved, which is the purpose of this section.

\section{Pitchwise averaged data}

The inlet and outlet planes have positioned closely to the blade at the axial direction of - $20 \%$ and $113 \%$ midspan axial chord, respectively, where the zero axial position indicates the LE at midspan as shown in Figure 5a. Another investigations located at the spanwise locations of $20 \%, 50 \%$ and $90 \%$ measured from the hub are shown in Figure $\mathbf{5 b}$. The flow field will be surveyed at these five locations where significant 3-D results of the fluid mechanical behaviour can be achieved.

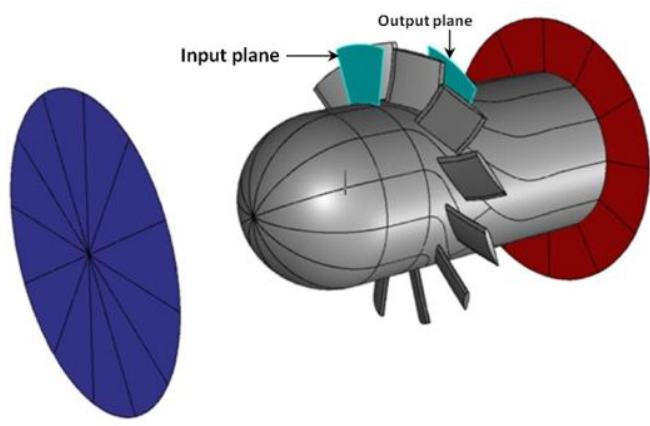

a) input and output planes

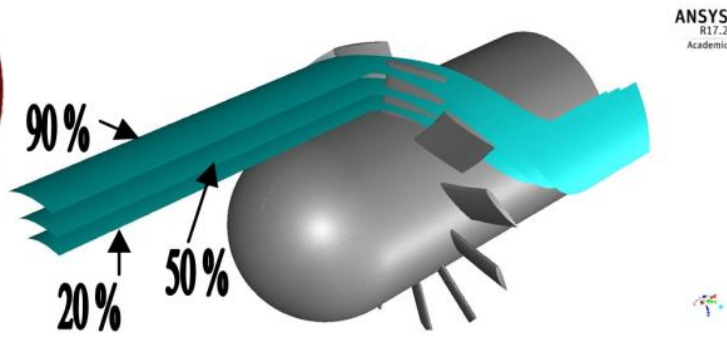

b) $20 \%, 50 \%$ and $90 \%$ span

Fig. 5. Location of results

The reference velocity $\left(u_{\mathrm{ref}}\right)$ or $\left(u_{\mathrm{t}}\right)$ in $[\mathrm{m} / \mathrm{s}]$ can be defined as blade tip speed $=\left(d_{\mathrm{t}} \pi n\right)$ where, $d_{t}$ is the blade tip diameter and $n$ is the rotor speed (in revolutions per second). The definition of local flow coefficient $(\varphi)$ is the ratio of axial velocity $\left(v_{x}\right)$ to $u_{\mathrm{t}}$

Figure 6a shows the distribution of local inlet axial velocity at the inlet plane and Figure $\mathbf{6 b}$ presents the spanwise distribution of pitchwise-averaged values for outlet axial velocity. Figure $\mathbf{6 b}$ indicates the two rotors having nearly similar pitch-averaged outlet axial velocity performance; which is possibly due to the small sweep angle. It was observed, that in Figure 6a, PSW decreases inlet axial velocity near the tip at PS, this acts to reduce the axial velocity downstream of the inlet especially at pressure side (PS) and blade LE as it is visible on the Figure 7. 


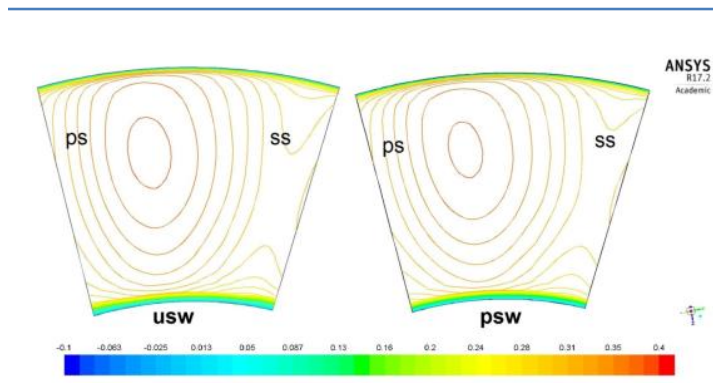

a) distribution of inlet $\varphi$

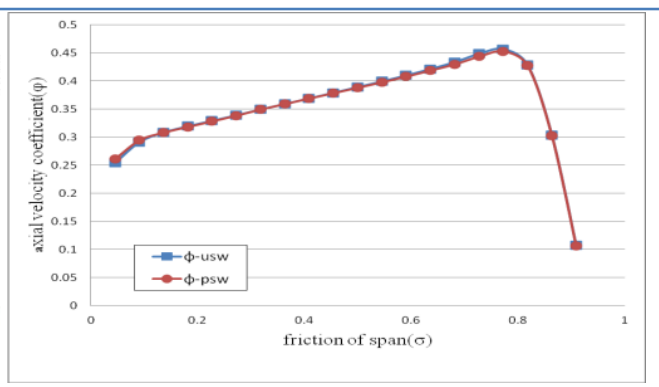

b) pitchwise-averaged outlet $\varphi$

Fig. 6. Inlet and outlet of axial velocity coefficients.

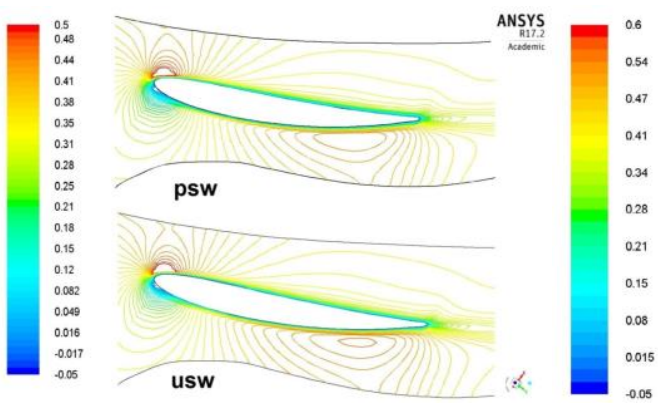

at $20 \%$ span

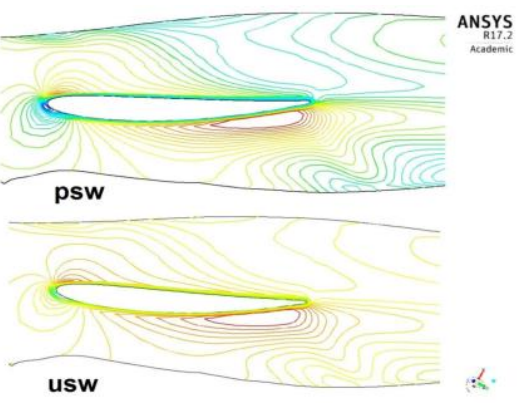

at $90 \%$ span

Fig. 7. Distribution of local axial velocity coefficient

The definition of ideal total pressure rise coefficient $\left(\psi_{i d}\right)$ is $\psi_{i d}=\Delta p_{\mathrm{t}}$ id $/\left(\rho{u_{\mathrm{ref}}}^{2} / 2\right)$. Where, $\Delta p_{\mathrm{t} \text { id }}=\rho r \omega \nu_{\mathrm{u} 2}, \nu_{\mathrm{u} 2}$ is the tangential pitchwise mass-averaged tangential velocity, $r$ is radial coordinate, $\omega$ is rotor angular speed and $\rho$ is the air density. For inviscid flow, the ideal total pressure rise $\left(\psi_{\text {id }}\right)$ of turbomachines is assumed swirl-free inlet flow according to the Euler equation. Also according to effects of the so called "controlled vortex design (CVD)" in both rotors, the ideal total pressure rises gradually increased along the blade spanwise especially above midspan, the ideal total pressure rise increases significantly at TE of the PSW which is due to the blade positivity, as visibly shown in Figure 8a. As zhang et al.[10] concluded that, the pressure fluctuation of forward sweep blade is decreased while the pressure fluctuation of backward sweep blade is increased, likewise to our case that, PSW blade tip acts to increase ideal total pressure rise near the cassing compared to $\mathbf{U S W}$ as shown in Figure $\mathbf{8 b}$, this is due to decreasing the axial velocity at higher radii especially at the blade pressure side. However, PSW reduces ideal total pressure compared to USW along the spanwise except near the blade tip. 


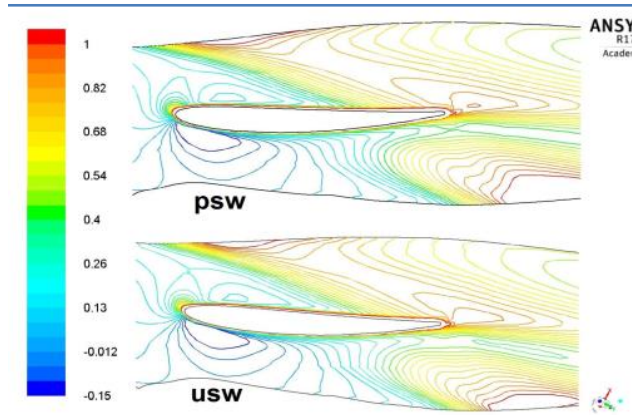

a) at $90 \%$ span

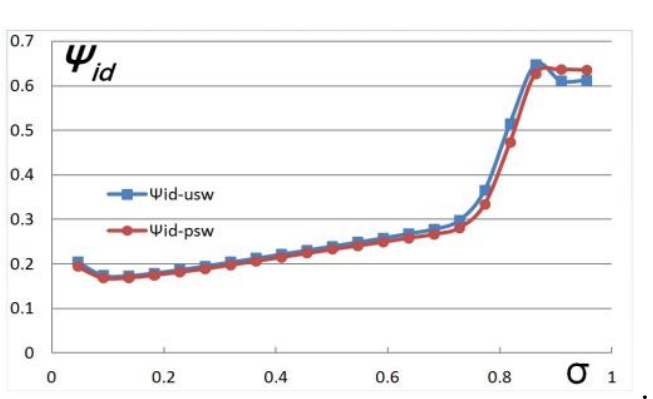

b) at span wise distributions

Fig. 8. Ideal total pressure rise coefficient

The definition of the local total pressure rise coefficient ( $\psi)$ is $\Delta p_{\mathrm{t}} /\left(\rho u_{\mathrm{ref}}{ }^{2} / 2\right)$, where $\Delta p_{\mathrm{t}}$ is the pitchwise mass-averaged local total pressure rise. The local total efficiency $(\eta)$ and total pressure loss coefficient $(\omega)$ can be defined as $\eta=\psi / \psi_{i d}$ and $\omega=\psi_{i d}-\psi$, respectively. Figure $9 \mathbf{b}$ shows the local total efficiency $(\eta)$ profiles along the span. By employing the positivity of sweep, the total local efficiency is observed to be decreased compared to the unswept rotor. PSW blade sweep showed reduced local efficiency especially at lower the midspan and close to the blade tip, this is due to the increased ideal total pressure rise and increased losses where the aerodynamic benefits of blade positivity would be expected on the basis of Clemen and Stark [4]. Furthermore, the local total efficiency is found almost similar to USW within the range of $70 \%$ to $85 \%$ of the spanwise otherwise. PSW exhibits the worst local total efficiency, especially at the part of the blade tip in suction side (SS) as shown in Figure9a.

It is pointed out that the local efficiency for PSW rotor is deteriorated near the tip which considered as a disadvantage of applying positive blade rotor at the case of design flow rate.

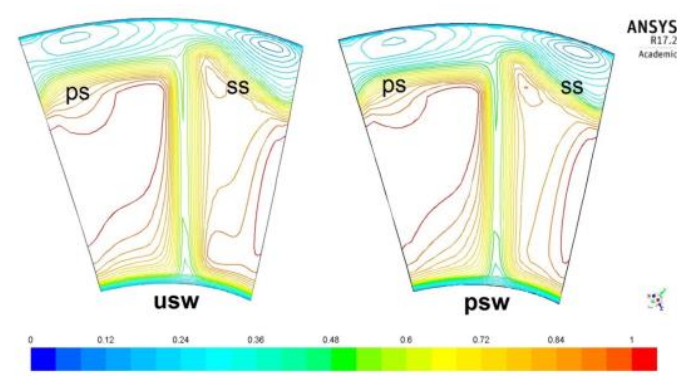

a) at outlet plane

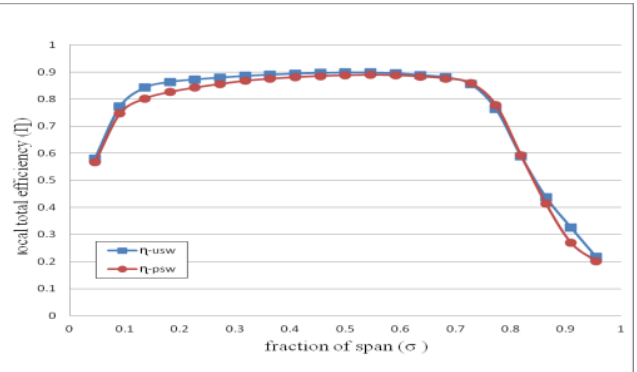

b) at span wise distributions

Fig. 9. Local total efficiency.

Increased total pressure loss for both rotors was found for both rotors above 80 percent span due to the existing tip clearance. At the blade tip, developing the local losses can be exist, the reason is that due to the combined effects of endwall tip-clearance, re-arrangement of the axial velocity and increased ideal total pressure rise, and can further increase by existing of positive

Proceedings of First Conference for Engineering Sciences and Technology (CEST-2018), vol. 2 
Aerodynamic Effects of Blade Positive Sweep in Axial Flow Cascades

sweep near the endwalls especially at SS, as shown in Figure 10. Clemen and Stark mentioned that, increased losses can be expected near the endwall in the case of negative sweep, due to opposite tendencies. By this means negative sweep and positive sweep can cause the shift of blade load toward the $\mathbf{L E}$ and toward the TE near the endwall, respectively [4]. By applying the positivity of sweep, the local total pressure loss observed to be increased at the blade tip, this causes to increase losses and decrease local total efficiency at the endwall.

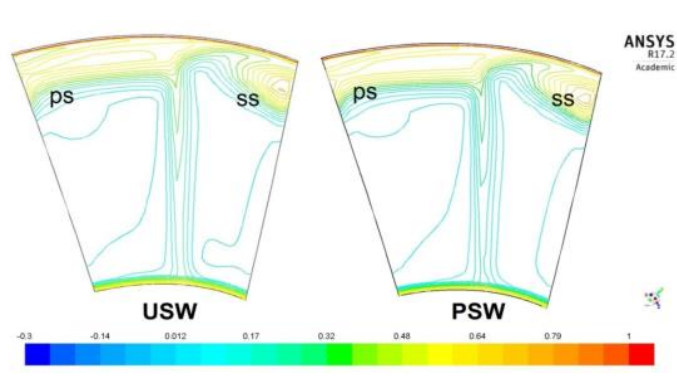

a) at outlet plane

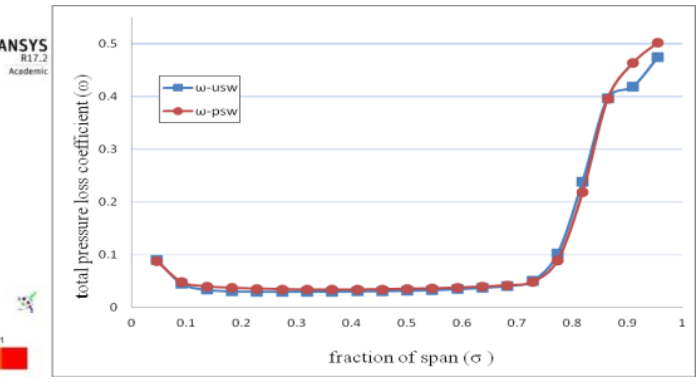

b) at span wise distributions

Fig. 10. Total pressure loss coefficient

\section{Conclusions}

The aim of this study was to investigate the effects of positive sweep applied to rotor of axial flow turbomachines using CFD tools. In this work, it is concluded that, the PSW rotor exhibits the lowest local total efficiency along the entire span, especially near the blade tip and near the hub at the design point, where as USW exhibits the highest local total efficiency along the entire span. It is pointed out generally that positive sweep gives a potential near-tip to increase of losses.

These results, therefore, demonstrate that the PSW rotor is considered as a disadvantage of applying positive blade rotor at the design flow rate.

Since, there is a deterioration of efficiency by using positive sweep near the tip and near the hub. It is recommended that, other investigations on a negative sweep should be carried out.

\section{References}

[1] Van Den Braembussche, R. A. and Vad, J. "Challenges in optimisation of axial flow turbomachinery blades for 3D flow, including sweep and dihedral effects. In Modelling Fluid Flow", Springer Verlag Heidelberg, 2004, pp. 99-103.

[2] Yamaguchi, N., Tominaga, T., Hattori, S., and Mitsuhashi, T., "Secondary-Loss Reduction by Forward-Skewing of Axial Compressor Rotor Blading," Proceedings Yokohama International Gas Turbine Congress, Yokohama, Japan, 1991,pp. II.61-II.68.

[3] Clemen, C., Gümmer, V., Goller, M., Rohkamm, H., Stark, U. and Saathoff, H., "Tip-aerodynamics of forward-swept rotor blades in a highly-loaded single-stage axial-flow low-speed compressor." 10th International Symposium on Transport Phenomena and Dynamics of Rotating Machinery (ISROMAC10), Honolulu, 2004, Paper No. 027. (CDROM Proceedings) https://www.researchgate.net/publication/274636618_Tipaerodynamics_of_forward_swept_rotor_blades_in_a_highly-loaded_single-stage_axial-flow_low-speed_compressor

[4] Clemen, C. and Stark, U. "Compressor blades with sweep and dihedral: a parameter study". 5th European Conference on Turbomachinery Fluid Dynamics and Thermodynamics, Prague, 2003, Proceedings pp. 151-161. 
[5] Corsini, A. and Rispoli, F., "Using Sweep to Extend the Stall-Free Operational Range in Axial Fan Rotors", Proc. Instn Mech. Engrs, Part A, J Power and Energy, 2004, Vol. 218, pp. 129-139.

[6] Vad, J., Kwedikha, A. R. A., Horváth, Cs., Balczó, M., Lohász, M. M., and Régert, T., "Combined Aerodynamic Effects of Controlled Vortex Design and Forward Blade Skew in Axial Flow Rotors". Proceedings of the Institution of Mechanical Engineers - Part A: Journal of Power and Energy, 2007, Vol. 221, pp. 1011-1023.

[7] Wenneker, Ivo, " Computation of flows using unstructured staggered grids" Dissertation at Delft University of Technology. 2002, Delft, the Netherlands.

[8] Rupak Biswas, Roger C. Strawn, "A dynamic mesh adaption procedure for unstructured hexahedral grids." Paper 960027, 34th AIAA Aerospace Sciences Meeting, Reno, NV, 1996.

[9] Kamenik, Jan, Keane, Andrew, Toal, David and Bates, Ron, “Application of sweep to transonic compressor rotor blades for low-order statistical moment averaging in robust design”. In Proceedings of the 1st Global Power and Propulsion Forum: GPPF, 2017, 7 pp.

https://eprints.soton.ac.uk/406744/

[10] Di Zhang, Jiao-Bin MA, and Qi Jing, "Numerical Study Of Unsteady Flow And Exciting Force For Swept Turbomachinery Blades”. Thermal Science, year 2016, vol. 20, suppl. 3, pp. s669-s676.

http://thermalscience.vinca.rs/pdfs/papers-2016/tsci160205199z.pdf 